\title{
High-Q hybrid 3D-2D slab-3D photonic crystal microcavity
}

\author{
Lingling Tang ${ }^{1, *}$ and Tomoyuki Yoshie ${ }^{1,2}$ \\ ${ }^{1}$ Department of Electrical and Computer Engineering, Fitzpatrick Institute for Photonics, \\ Duke University, Durham, North Carolina 27708-0291, USA \\ ${ }^{2}$ e-mail: yoshie@duke.edu \\ ${ }^{*}$ Corresponding author: lingling.tang@duke.edu
}

Received June 18, 2010; revised August 20, 2010; accepted August 27, 2010; posted August 30, 2010 (Doc. ID 130384); published September 15, 2010

\begin{abstract}
The radiation loss in the escaping light cone with a two-dimensional (2D) photonic crystal slab microcavity can be suppressed by means of cladding the low- $Q$ slab microcavity by three-dimensional woodpile photonic crystals with the complete bandgap when the resonance frequency is located inside the complete bandgap. It is confirmed that the hybrid microcavity based on a low- $Q$, single-defect photonic crystal slab microcavity shows improvement of the $Q$ factor without affecting the mode volume and modal frequency. Whereas 2D slab microcavities exhibit $Q$ saturation with an increase in the number of layers, for the analyzed hybrid microcavities with a small gap between the slab and woodpiles, the $Q$ factor does not saturate. (C) 2010 Optical Society of America

OCIS codes: $230.5298,230.5750$
\end{abstract}

An extensive amount of research has been conducted in designing microcavities and waveguides based on a twodimensional (2D) photonic crystal slab, in which one fundamental limitation is the out-of-plane loss due to the imperfect confinement of light in total internal reflection in the direction perpendicular to the slab. Although simple 2D photonic crystal defect microcavities, such as a single-defect microcavity, can be designed without much computational resources, they tend to show low $Q$ factor, and the improvement of the $Q$ factor requires a substantial amount of design resources due to trial and error approaches. One way to improve the confinement is to reduce the field components inside the escaping light cone [1-3]; however, this method could only decrease, but not eliminate, the power radiation loss into the escaping light cone. As a result, even with sophisticated designs [4-8], the $Q$ factor of 2D slab microcavities could only be increased to some extent, and would still saturate even for finite photonic crystal unit cells. In addition, the mode volume is large for high- $Q$ microcavities [3-6]; i.e., one obtains high $Q$ factor at the expense of large mode volume, resulting in a limited choice in the mode volume. Another approach is to combine the slab microcavity with distributed Bragg reflectors [9,10]; however, this quasi-three-dimensional (3D) photonic crystal, that is, a one-dimensional (1D)-2D slab-1D hybrid microcavity, does not have omnidirectional optical confinement, and an ultrahigh $Q$ factor has not been demonstrated. On the other hand, a 3D photonic crystal with the complete bandgap provides 3D confinement. Chutinan and John sandwiched an intermediate layer with 3D photonic crystals for lossless 3D integrated optics [11]. Although there is remarkable progress in $3 \mathrm{D}$ photonic crystal fabrication $[12,13]$, only a few microcavity and waveguide designs are available in 3D photonic crystals [14-16]. On the contrary, various designs have been developed on 2D photonic crystal slabs, including microcavities [4-8] and straight and bent waveguides [17]. To recycle simple 2D slab designs and improve the vertical optical confinement without substantial computational resources, we propose to clad 2D slab nano-optical devices with 3D photonic crystals whose complete photonic bandgap overlaps with the mode frequency of the 2D slab optical components. In this work, we demonstrate the enhancement of the $Q$ factor by using a simple but low- $Q 2 \mathrm{D}$ microcavity design - a 2D slab triangular lattice photonic crystal missing a single hole, i.e., the so-called L1 cavity [18].

The L1 cavity exhibits small mode volume with low $Q$ factor, because a large portion of Fourier-transformed field component resides inside the escape light cone in the momentum space. The L1 cavity analyzed in this work has a slab thickness of $d=0.95 a_{2 \mathrm{D}}$ and a hole radius of $r=0.3 a_{2 \mathrm{D}}$, where $a_{2 \mathrm{D}}$ is the lattice constant of the $2 \mathrm{D}$ slab photonic crystal. The refractive index of the slab is 3.4. Figure 1(a) illustrates the structure of the L1 cavity. In the $3 \mathrm{D}$ finite-difference time-domain (FDTD) analysis [19], we selectively excite the $y$-dipole mode with a mode frequency of $0.271\left(c / a_{2 \mathrm{D}}\right)$, where $c$ is the velocity of light in vacuum. The field distributions are mapped in Figs. 1(b) and 1(c). The saturated $Q$ factor, which is determined by the radiation loss in the direction vertical to the slab, is as low as 205 [see data indicated by blue diamonds in Fig. 4(a) ], but the mode volume is as small as $2.9\left(\lambda_{0} / 2 n\right)^{3}$, where $\lambda_{0}$ is the mode wavelength in vacuum.

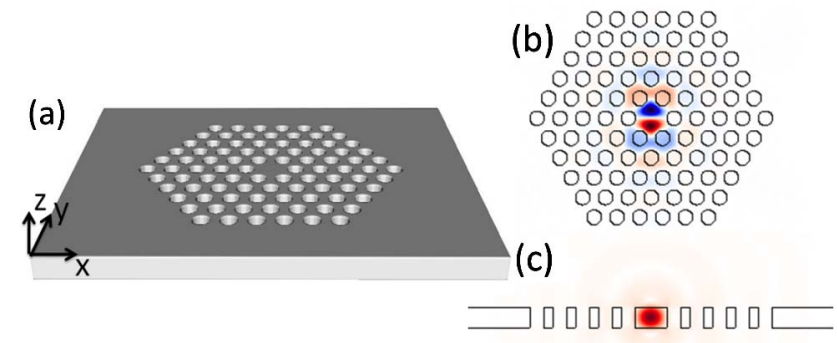

Fig. 1. (Color online) L1 cavity and the $y$-dipole mode. (a) Schematic of the L1 cavity without woodpile cladding. There are five layers in both the $x$ and $y$ directions for optical confinement. (b) $H_{z}$ distribution in the middle of the $x-y$ plane in the slab. (c) Amplitude of the electric field in the middle of the $x-z$ plane. 
Next, we place woodpile photonic crystals on the top and bottom surfaces of the slab; see Fig. 2(a) for the structure of the 3D-2D slab-3D hybrid microcavity. The dielectric material forming woodpile photonic crystals has the index $n=3.4$ as well. We choose the rod width $(w)$ and height $(h)$ of the woodpile photonic crystals as $w=$ $h=0.3 a_{\mathrm{wp}}$, where $a_{\mathrm{wp}}$ is the lattice constant of the woodpile photonic crystals, i.e., the center-to-center distance of the adjacent rods in the same layer. The complete photonic bandgap ranges between $0.35\left(c / a_{\mathrm{wp}}\right)$ and $0.43\left(c / a_{\mathrm{wp}}\right)$. For the frequency inside this bandgap, the optical field evanescently decays in all directions in the woodpile photonic crystal. We consider three design factors for the hybrid microcavities. First, we match the mode frequency with the midgap of the woodpile with an appropriate choice of the lattice constant $a_{\mathrm{wp}}$. Assuming little change in the mode frequency induced by placing the woodpile on the top and bottom surfaces of the L1 cavity slab, we choose $a_{\mathrm{wp}}=1.5 a_{2 \mathrm{D}}$, so that the mode frequency, which is about $0.271\left(c / a_{2 \mathrm{D}}\right)$, is inside the bandgap $\left(0.23\left(c / a_{2 \mathrm{D}}\right), 0.29\left(c / a_{2 \mathrm{D}}\right)\right)$. Second, we may choose an arbitrary crystal orientation of the woodpile photonic crystal relative to the $2 \mathrm{D}$ slab thanks to the omnidirectional bandgap. Here we choose the $x$ axis as the woodpile stacking direction. Third, we choose the gap size $(g)$ between the slab and the nearest surface of the woodpile photonic crystal. We vary the gap size and study the effect of the value $g$ on the performance of the 3D-2D slab-3D hybrid microcavity. The structure size is defined by the number of layers in the 2D slab in the $x$ and $y$ directions $\left(N_{x 2 \mathrm{D}}\right.$ and $\left.N_{y 2 \mathrm{D}}\right)$ and the number of unit cells of the woodpile on each side in the $x, y$, and $z$ directions $\left(N_{x \mathrm{wp}}, N_{y \mathrm{wp}}\right.$, and $\left.N_{z \mathrm{wp}}\right)$. In 3 D FDTD calculation, the structure size $N$ is chosen to be $N=N_{x 2 \mathrm{D}}=N_{y 2 \mathrm{D}}=N_{z \mathrm{wp}}$. Because the lattice constant of the 2D slab can differ from that of the woodpiles, the unit cell number does not necessarily match between the 2D slab and the woodpile in the $x$ or $y$ directions; instead, we choose $N_{x \mathrm{wp}}=\operatorname{Round}\left(a_{2 \mathrm{D}} \cdot N_{x \mathrm{D}} /(2 h)\right)$ and $N_{y \mathrm{wp}}=$ $\operatorname{Round}\left(\sqrt{ } 3 \cdot a_{2 \mathrm{D}} \cdot N_{y 2 \mathrm{D}} / a_{\mathrm{wp}}\right)$ so that the woodpile covers the $2 \mathrm{D}$ slab photonic crystal area.

We first study the effect of gap size between the slab and the woodpile on the $Q$ factor of the $y$-dipole mode. The mode frequency is slightly lowered as the gap size reduces, as shown in Fig. 3, but the mode symmetry is preserved. The mode frequency barely changes as the structure sizes are varied for each $g$ value. Figures $2(\mathrm{~b})$ and $2(\mathrm{c})$ show the $H_{z}$ component in the middle of the $\overline{x-y}$ plane and the amplitude of the electric field in the middle of the $x-z$ plane, respectively. Figure 4(a) shows the $Q$ factor of the hybrid microcavity as a function of structure size $N$ for different gap sizes. Except for small $N$ (e.g., $N=3$ ), the confinement apparently improves with woodpile photonic crystal cladding more than that without cladding (i.e., $g=\infty$ ). For $N=3$, the $Q$ factor is independent of the value $g$, because the radiation loss dominates in the planar directions of the slab, and the $Q$ factor is not significantly altered by the cladding woodpile. For the same $N$, as the value $g$ decreases, the $Q$ factor first increases due to reduced power loss through the gap. The $Q$ factor reaches a maximum value at $g=0.05 a_{2 \mathrm{D}}$ and decreases slightly as we further reduce the gap size to 0, i.e., the woodpile photonic crystals touch the slab. This is probably because the perturbation is stronger for $g=0$ than

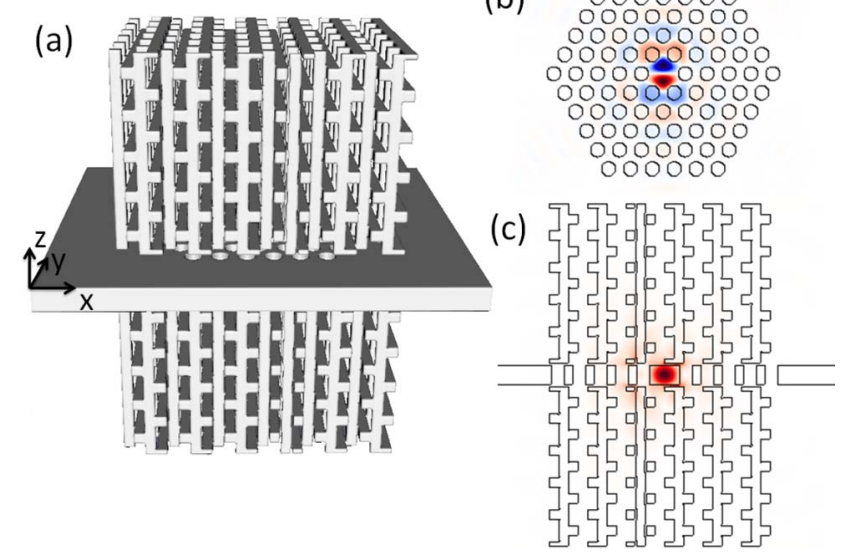

Fig. 2. (Color online) Hybrid microcavity and the $y$-dipole mode. (a) Schematic of the hybrid microcavity constructed by cladding the L1 cavity with woodpile photonic crystals on the top and bottom surfaces of the slab. The woodpiles are stacked along the $x$ direction. There are five layers in the slab. Each woodpile photonic crystal has $6 \times 6 \times 5$ unit cells. The gap size $g$ is $0.05 a_{2 \mathrm{D}}$. (b) $H_{z}$ distribution in the middle of the $x-y$ plane in the slab. (c) The amplitude of the electric field in the middle of the $x-z$ plane.

for $g=0.05 a_{2 \mathrm{D}}$. For a large value of $g$, the $Q$ factor saturates as $N$ increases, such as in the case of $g=a_{2 \mathrm{D}}$ [see green triangles in Fig. 4(a)] because of the coupling with the guided modes supported in the large gap case. On the other hand, if the gap size is sufficiently small, the $Q$ factor exponentially increases without any sign of saturation as the unit cell number $N$ increases. This is evidence of optical confinement owing to the complete photonic bandgap. For both the $g=0$ (black squares) and $g=0.05 a_{2 \mathrm{D}}$ (red circles) cases, the $Q$ factor can exceed $10^{5}$, so the hybrid microcavity is robust in terms of the gap size in the analyzed range. The hybrid microcavity also preserves the small mode volume: $3.0\left(\lambda_{0} / 2 n\right)^{3}$ for both $g=0$ and $g=0.05 a_{2 \mathrm{D}}$; see Fig. 4(b). Thus, a high Q factor with a similar mode volume is demonstrated. Even a large gap example $\left(g=a_{2 \mathrm{D}}\right)$ exhibits $Q$ improvement.

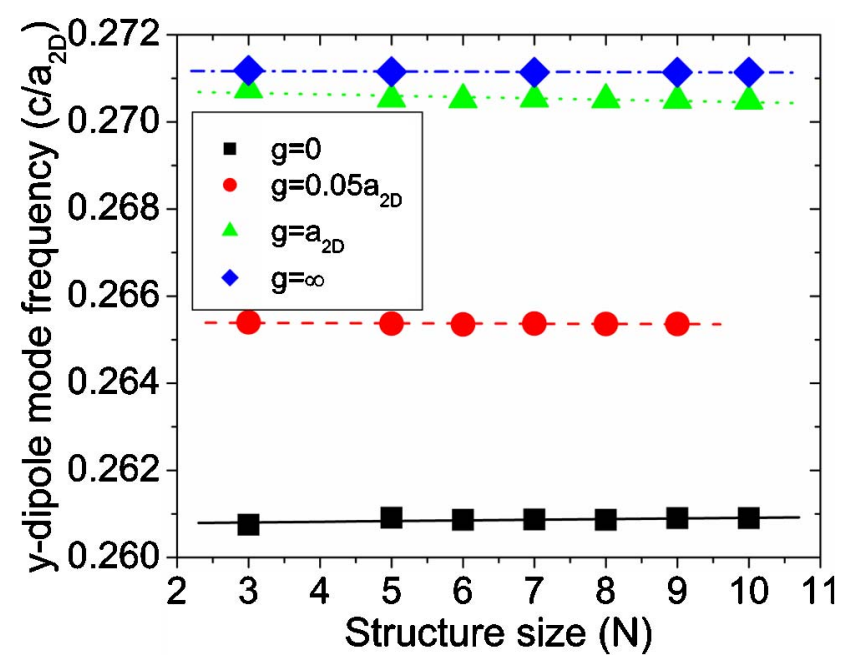

Fig. 3. (Color online) The $y$-dipole mode frequency as a function of structure sizes $\left(N=N_{x 2 \mathrm{D}}=N_{y 2 \mathrm{D}}=N_{z \mathrm{wp}}\right)$ for different gap values. The frequency is reduced by $3.7 \%$ for $g=0$ compared to $g=\infty$. 
(a)

(b)
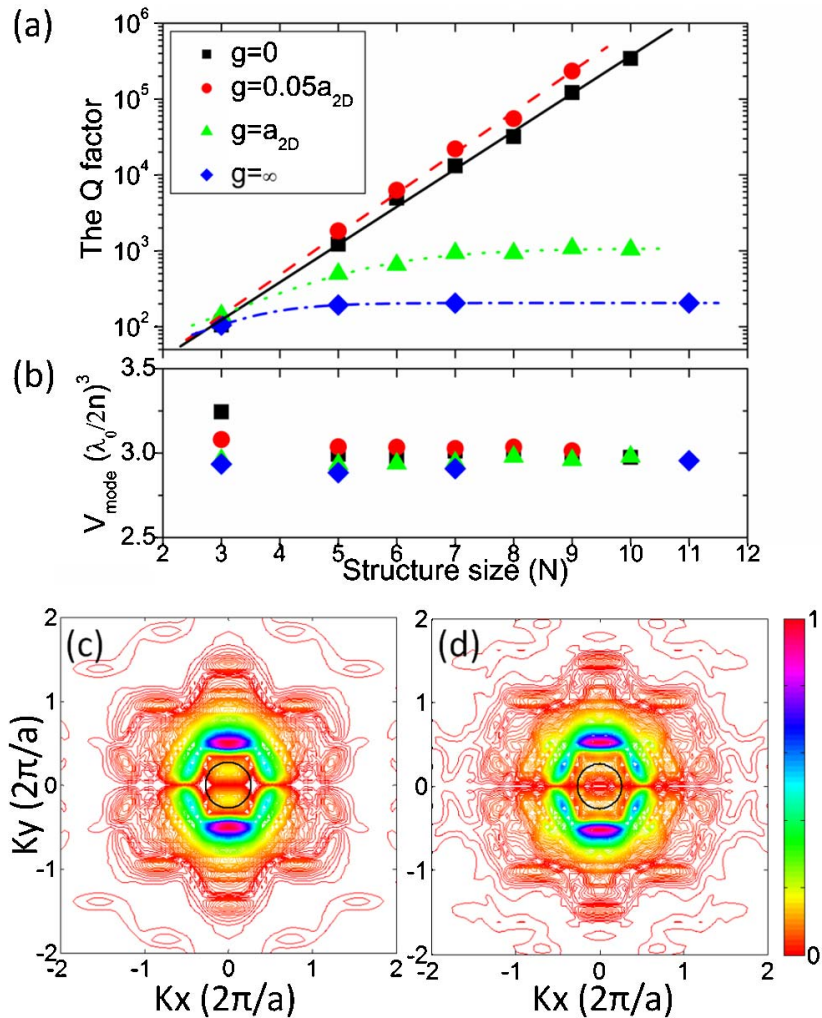

Fig. 4. (Color online) Characteristics of the hybrid $(g<\infty)$ and L1 $(g=\infty)$ microcavities, and Fourier space mapping of the $H_{z}$ component. (a) $Q$ factor and (b) the mode volume for different gap and structure sizes. The structure size is $N=$ $N_{x 2 \mathrm{D}}=N_{y 2 \mathrm{D}}=N_{z \mathrm{wp}}$. (c) Fourier-transformed $H_{z}$ component near the surface of the L1 cavity. The black circle indicates the light cone. (d) Fourier-transformed $H_{z}$ component of the hybrid cavity with $g=0.05 a_{2 \mathrm{D}}$.

To examine the origin of the $Q$ improvement, we calculate the Fourier-transformed $H_{z}$ component near the surface of the slab for both $g=\infty$ and $g=0.05 a_{2 \mathrm{D}}$, in Figs. 4(c) and 4(d), respectively. The scattering is slightly enhanced for $\bar{g}=0.05 a_{2 \mathrm{D}}$, but the overall pictures are similar, and there are no significant differences in the sum of the Fourier components inside the escaping light cone. Therefore, we understand that the $Q$ factor is enhanced by the complete photonic bandgap, not by removing components from the inner region of the escaping light cone.

In conclusion, the radiation loss due to failure of total internal reflection in a slab can be suppressed via the sandwiching of a $2 \mathrm{D}$ slab with $3 \mathrm{D}$ photonic crystals. By cladding woodpile photonic crystals onto the top and bottom surfaces of an L1 cavity, we can increase the
$Q$ factor from 205 to more than $10^{5}$ without a complicated redesign, thanks to the complete photonic bandgap. We believe that this method is applicable to other lossy 2D slab photonic crystal microcavity and waveguide designs to easily improve the optical confinement while maintaining the mode properties, such as mode symmetry and mode volume, as long as the modes are not significantly disturbed by the introduction of 3D photonic crystals. The power losses of integrated optics components based on 2D photonic crystal slabs would be reduced. Therefore, the proposed hybrid structures would achieve low-loss integrated optics without extra efforts in designing and integrating 3D photonic crystal microcavities and waveguides.

The authors acknowledge the National Science Foundation (NSF) (ECCS-0901599) for financial support.

\section{References}

1. K. Srinivasan and O. Painter, Opt. Express 10, 670 (2002).

2. J. Vuckovic, M. Loncar, H. Mabuchi, and A. Scherer, IEEE J. Quantum Electron. 38, 850 (2002).

3. D. Englund, I. Fushman, and J. Vuckovic, Opt. Express 13, 5961 (2005).

4. Y. Akahane, T. Asano, B.-S. Song, and S. Noda, Nature 425, 944 (2003).

5. B.-S. Song, S. Noda, T. Asano, and Y. Akahane, Nat. Mater. 4, 207 (2005).

6. E. Kuramochi, M. Notomi, S. Mitsugi, A. Shinya, T. Tanabe, and T. Watanabe, Appl. Phys. Lett. 88, 041112 (2006).

7. K. Nozaki and T. Baba, Appl. Phys. Lett. 88, 211101 (2006).

8. K. Nozaki, S. Kita, and T. Baba, Opt. Express 15, 7506 (2007).

9. N. Susa, Jpn. J. Appl. Phys. 42, 7157 (2003).

10. M. Ito, S. Iwamoto, and Y. Arakawa, Jpn. J. Appl. Phys. 43, 1990 (2004).

11. A. Chutinan and S. John, Opt. Express 14, 1266 (2006).

12. A. Tandaechanurat, S. Ishida, K. Aoki, D. Guimard, M. Nomura, S. Iwamoto, and Y. Arakawa, Appl. Phys. Lett. 94, 171115 (2009).

13. K. Ishizaki and S. Noda, Nature 460, 367 (2009).

14. M. Okano, S. Kako, and S. Noda, Phys. Rev. B 68, 235110 (2003).

15. L. Tang and T. Yoshie, Opt. Express 15, 17254 (2007).

16. S. Kawashima, L. H. Lee, M. Okano, M. Imada, and S. Noda, Opt. Express 13, 9774 (2005).

17. A. Chutinan and S. Noda, Phys. Rev. B 62, 4488 (2000).

18. O. Painter, J. Vuckovic, and A. Scherer, J. Opt. Soc. Am. B 16, 275 (1999).

19. A. Farjadpour, D. Roundy, A. Rodriguez, M. Ibanescu, P. Bermel, J. D. Joannopoulos, S. G. Johnson, and G. Burr, Opt. Lett. 31, 2972 (2006). 